\title{
REFLEXIONES SOBRE LA ENSEÑANZA DE LA ARQUITECTURA
}

\author{
Jorge Luis Poma García*
}

\section{EL PROCESO DE DISEÑO ARQUITECTÓNICO}

Incluso si admitimos que la arquitectura tiene en común con la ciencia la búsqueda de una solución a un problema, no debemos perder de vista el hecho de que la concepción arquitectónica se encuentra situada en la concurrencia de las artes y las ciencias. La arquitectura tiene la especificidad de responder a un problema que generalmente no está completamente formulado y cuyos modos de resolución y resultados son múltiples. Uno se siente tentado a pensar que existen tantos modos de gestión del proceso de concepción como arquitectos existen; sin embargo, los diversos estudios realizados sobre el análisis del proceso de diseño arquitectónico muestran que se pueden agrupar enfoques estándar a partir de los cuales son posibles las estrategias personales.

Las referencias a la historia nos recuerdan que a fines del siglo $X V$, cuando se estructura el primer discurso sobre la arquitectura, aparecen los crite- rios de definición comunes a cualquier proyecto arquitectónico. Leon Battista Alberti define la arquitectura como una "cosa mental», cuyo objeto es el arte de la vida social que se basa en los criterios de "necessitas, commoditas, voluptas». La necesidad se entiende aquí como la dependencia de la construcción de las leyes físicas y mecánicas (técnica). La comodidad se expresa por la demanda social (usos). La voluptuosidad se define como la capacidad de la arquitectura para significar por su propia cuenta una poética declarada por un lenguaje arquitectónico proporcionando un placer derivado de la sensación de belleza (estética). Finalmente, según Alberti, la calidad del diseño depende de las "concinnitas", que es la armonía y la coherencia entre estos diversos criterios. Esta trilogía ha sido reformulada, renombrada, matizada, pero jamás desmentida durante siglos, y los arquitectos reconocemos fácilmente en esta definición la especificidad de nuestra actividad.

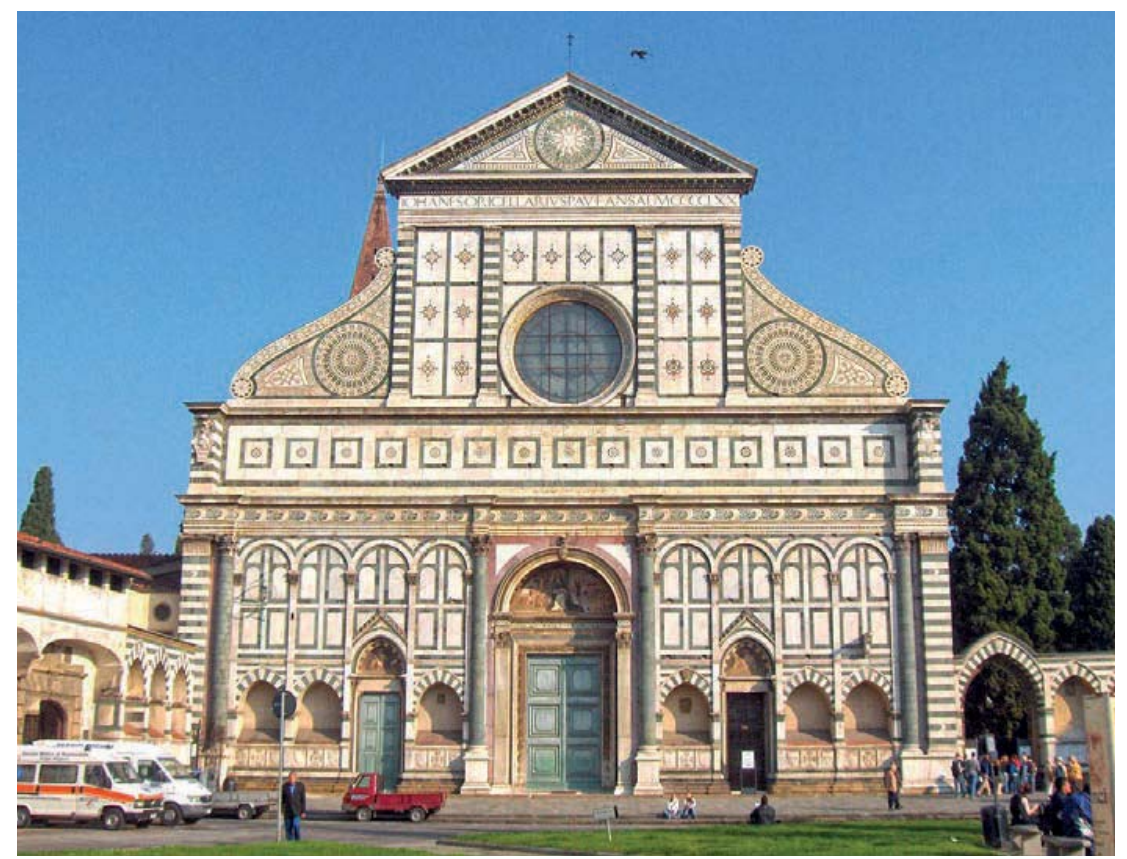

Figura 1. Basilica Santa Maria Nouvelle, FlorenciaItalia. Fachada obra de Leon Battista Alberti 1470.

Fuente: https://commons. wikimedia.org

* Maestro en Ciencias del Medio Ambiente, especialidad Ecología Urbana, Universidad de Ginebra. Docente de la EAP Arquitectura, Universidad Continental. 


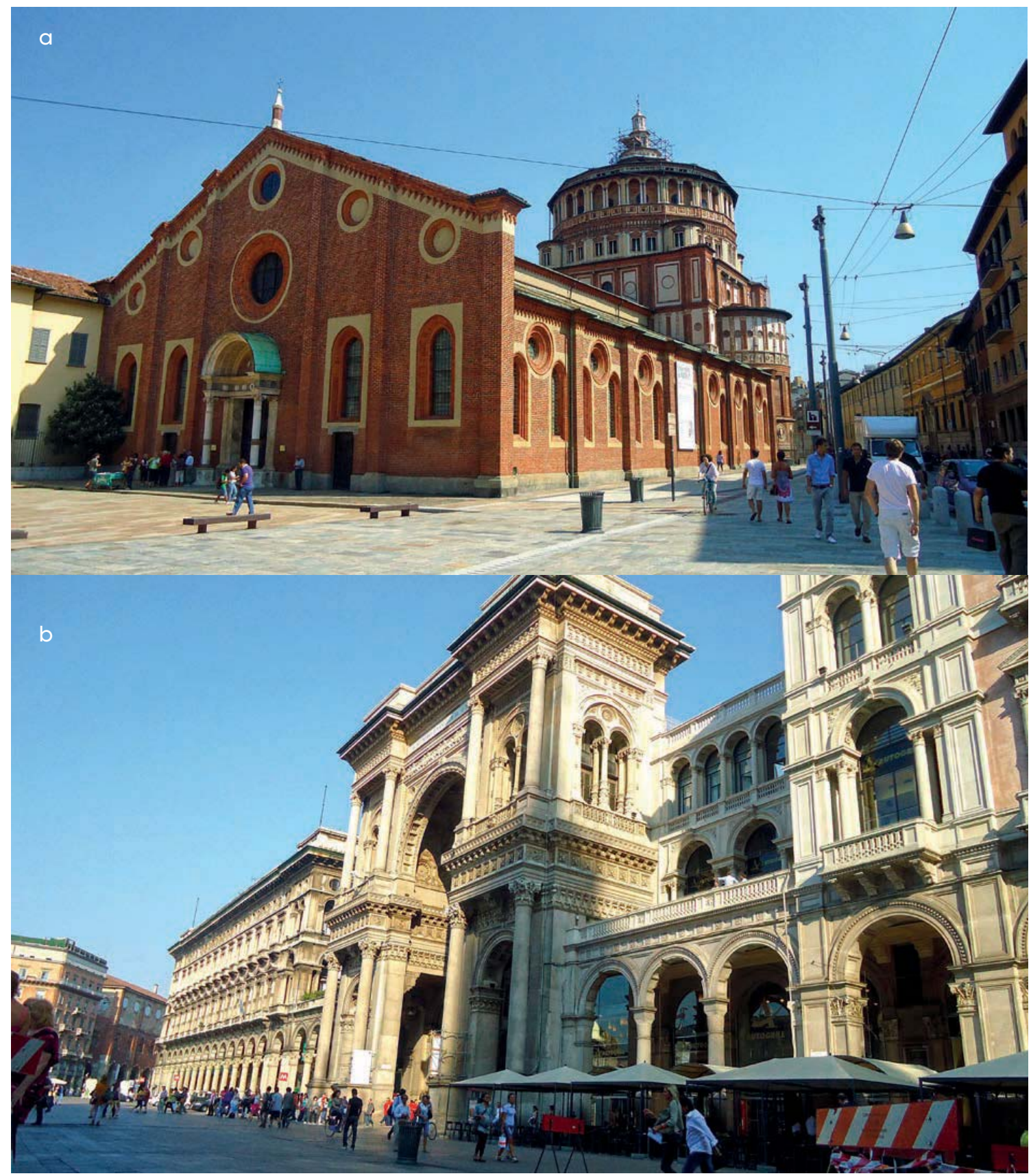

Figura 2. Fotos de arquitectura gótica, renacentista y neorrenacentista. Milán, Italia.

a) Iglesia Santa Maria delle Grazie, obra del arquitecto Donato Bramante, que forma parte del convento de la Orden de los Hermanos Predicadores. En esta iglesia se encuentra La última cena, de Leonardo da Vinci.

b) Galería de Victor Manuel II, obra del arquitecto Giuseppe Mengoni, ubicada al lado norte de la Piazza del Duomo en Milán.

Fuente: Jorge Luis Poma García 
Sin embargo, estos criterios que interactúan tanto en el nivel global de la obra (el proyecto) como en el nivel de los componentes deben darse simultáneamente $\mathrm{y}$, por lo tanto, hacen que sea difícil la modelización del proceso de concepción.

Los enfoques de los arquitectos, diseñadores, se diferencian precisamente por la importancia que otorgarán a los diversos criterios. Esta importancia de hecho condiciona la jerarquía de integración de restricciones en el proceso de concepción. Esta jerarquización de las restricciones que tendrán lugar, de manera consciente o no, durante el proceso es, particularmente, determinante para permitir que el diseñador formalice un punto de vista inicial. Por esta razón, los primeros trabajos de investigación sobre el modelado del proceso de concepción arquitectónica se centraron en cómo iniciar este proce- so. A partir de estos trabajos sobre los enfoques metodológicos, es que generalmente podemos referirnos al enfoque de los arquitectos a una de las siguientes dos familias de pensamiento: El empirismo induccionista o los procesos heurísticos, cada una de los cuales puede dar lugar a diferentes modelos de concepción.

\section{Enfoques metodológicos}

El empirismo induccionista es una corriente de pensamiento que parte de las reflexiones del Círculo de Viena'. Se caracteriza por la certeza de que cualquier dificultad para resolver un problema radica en la capacidad de plantearlo correctamente. Esto implica que un análisis bien realizado permite realizar observaciones perspicaces de las suposiciones que deberán confirmarse por pruebas empíricas.


Figura 3. Concurso Estructurando 2018-20. Experimentacion de los estudiantes.

Foto: Carol Alvarez Cortez

\footnotetext{
1 Grupo conformado por científicos y filósofos, fundado en Viena por Moritz Schlick, filósofo austríaco. Este movimiento desarrolla el concepto unificado de la "concepción científica del mundo"; se ocupaba, fundamentalmente de la lógica de la ciencia y de la construcción de un lenguaje único a todas las ciencias -la filosofía era la disciplina encargada de dilucidad entre lo qué es ciencia y o que no lo era-.
} 
Del empirismo induccionista, se desprenden los siguientes modelos de concepción:

- La programación arquitectónica, es un enfoque que consiste en un trabajo muy detallado del diseñador asociado a la gestión de proyectos que involucra las fases de: elaboración sumaria, programación fina y ejecución.

- La sintesis de la forma es un método que consiste en acercarse a la fase de análisis para dividir el problema en "subproblemas" que son más fáciles de dominar por ensambles sucesivos hasta el reagrupamiento final en un solo problema.

- La arquitectura conceptual se basa en la relevancia del análisis, pero otorga una mayor participación a las intuiciones. La invención, proceso de concepción, se basa en la aparición de una «idea» después del análisis que se puede asimilar a una sintesis inconsciente que involucra las fases de: problema, suposiciones, exploración, incubación y filtro, según los criterios del espíritu, invención y verificación.

Los procesos heurísticos, o metodología heurística, es una corriente de pensamiento que otorga mayor jerarquía a la intuición que al análisis para resolver un problema. Karl Popper ${ }^{2}$ afirma que ninguna decisión no puede ser deducida de un enunciado de hecho; por el contrario, sostiene que la mente, dotada de un conocimiento previo, construye las mismas observaciones que son luego comparadas con el problema planteado.

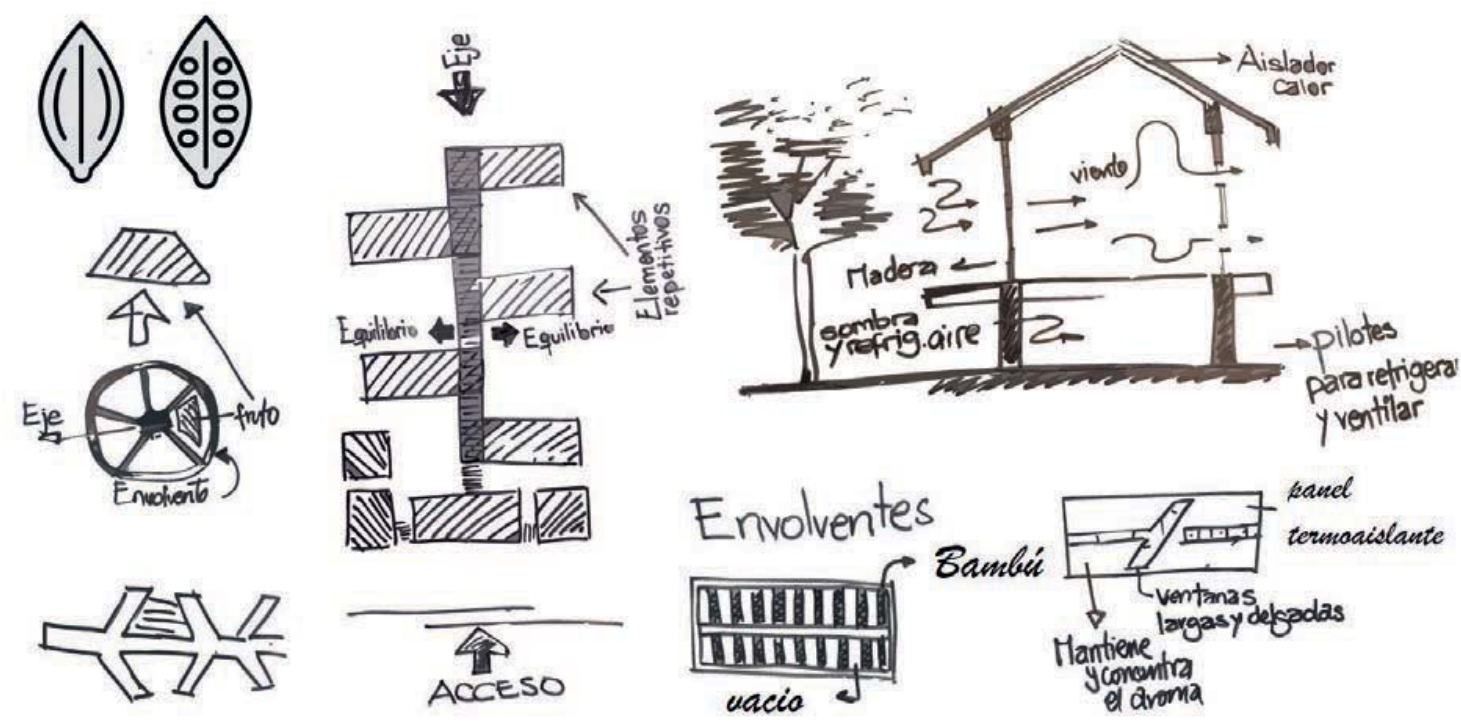

Figura 4. Criterios arquitectónicos aplicados al proyecto.

Foto: Kathia Velasque Vasquez

2 Fundador de la corriente epistemológica del falsacionismo, principio de falsabilidad o racionalismo crítico. Para Popper, corroborar la universalidad de una teoría es un procedimiento en el cual se trata de contrastarla, refutarla, mediante un contraejemplo. Si esto no es posible, dicha teoría queda aceptada provisionalmente, pero no verificada, es decir, ninguna teoria es absolutamente verdadera, sino a lo sumo "refutada". El falsacionismo de Popper es considerado hasta hoy como uno de los pilares del método científico. 
Esta corriente de pensamiento ha dado lugar a una reflexión sobre los modelos de concepción arquitectónica siguientes:

- El modelo de aprendizaje considera que la concepción es el fruto de la confrontación del contexto y de una imagen objetivo, formulada intuitivamente por el diseñador: imagen/formalización/ y producto final.

- El método de integración interactiva se basa en el principio de que toda la dificultad de la concepción consiste en pasar del análisis a la formalización. Por lo tanto, este método proscribe cualquier intento de análisis exhaustivo y recomienda, por el contrario, liderar al mismo tiempo el análisis y la propuesta.
Por lo tanto, cada elemento de observación está asociado con una forma global de respuesta que se desafía constantemente hasta que el problema se explora por completo.

- El enfoque tipológico se basa en la afirmación de que este es el tipo de orden que induce el proceso a:

- la realización de objetos arquitectónicos con un componente simbólico fuertemente ligado a la "intuición» de creador y a su bagage cultural.

- la realización de un sistema singular de organización (respuesta a un programa original) asume que el programa es parte fundamental del proceso de diseño, el cual está ligado íntimamente a los usuarios.

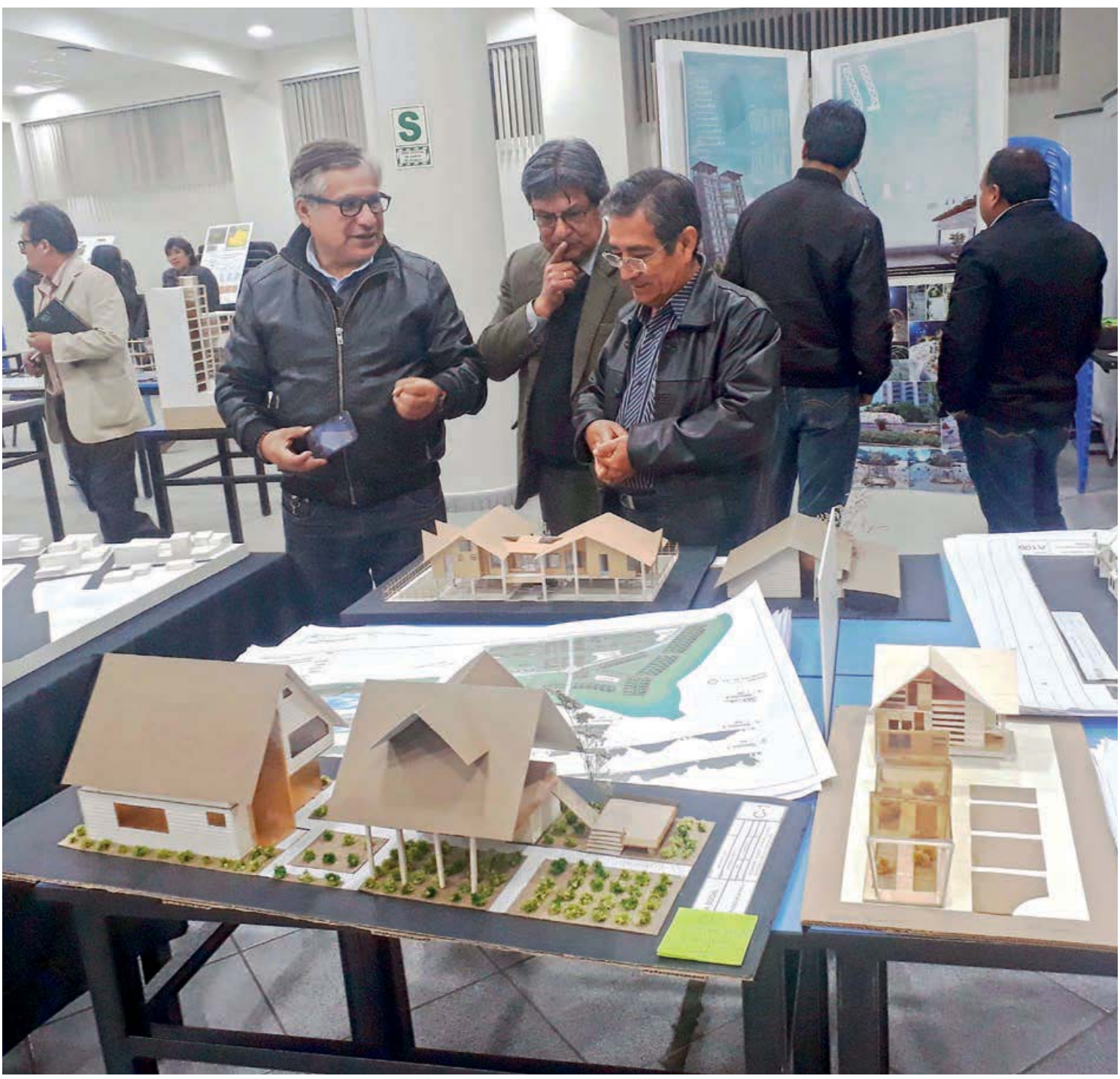

Figura 5. Docentes de la Escuela Académico Profesional Arquitectura en la Expo-Arquitectura 2018-10. Foto: Jorge Luis Poma García 


\section{EL PROYECTO DE ARQUITECTURA Y SU ENSEÑANZA}

La enseñanza de la arquitectura del proyecto se lleva a cabo en las escuelas, en los talleres educativos, lugares y tiempo dedicados exclusivamente a la práctica del diseño y están directamente relacionados con la práctica del proyecto o cuestiones derivadas de ejercicios específicos (Lebahar, 2001).

La enseñanza del proyecto en arquitectura es una situación artificial de diseño, finalizado por la realización de un estado de competencias del estudiante.

La Figura 6 ayuda a identificar los principales aspectos de una situación didáctica de proyecto: "hay situación didáctica cada vez que podemos caracterizar una intención de enseñanza de un saber por un profesor a un estudiante, y qué mecanismos socialmente definidos son instituidos para ello» (Johsua \& Dupin, 1993).

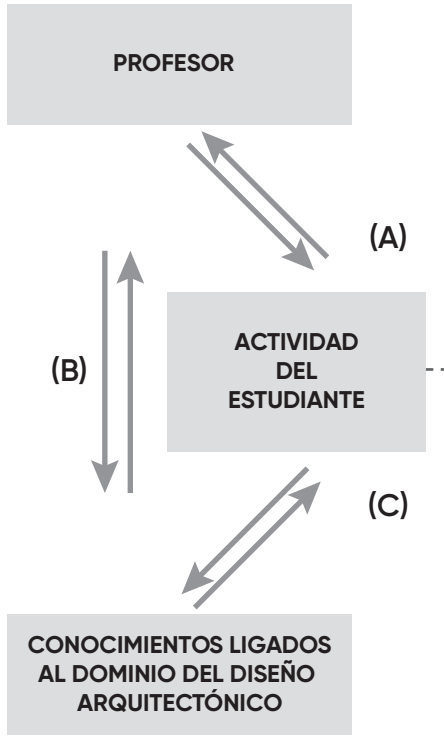

\section{INICIO DEL EJERCICIO}

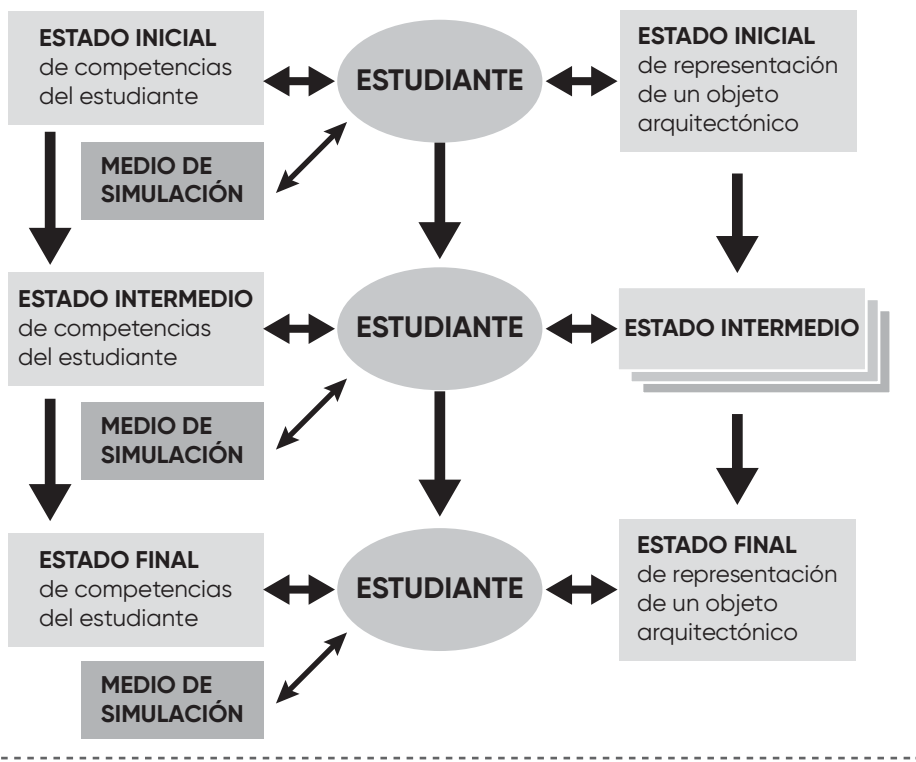

Figura 6. La enseñanza del proyecto en arquitectura.

Fuente: Lebahar J., 2001.

La interacción (A) vincula al profesor con «la actividad del estudiante» de diferentes maneras:

- El profesor construye la situación que enfrenta al estudiante a una tarea de diseño (transformar "el estado inicial de la representación de un objeto arquitectónico" con la ayuda de «medios de simulación" bien definidos).

- El profesor fija los objetivos y las condiciones para la realización de esta tarea (resultados esperados, la duración del ejercicio, fechas y de las modalidades de corrección).
- El profesor determina cómo son dadas las instrucciones a los estudiantes para la realización de ejercicios (en bloque o sucesivas subtareas).

El profesor tiene como objetivo llevar a los estudiantes a un "estado final de competencias". Para lograr este objetivo, deben adaptarse tareas, las cuales son presentadas a los estudiantes en los diferentes niveles y áreas de especialización. A menudo, el maestro utiliza mucho la variable tiempo como un acelerador o retardador para las actividades de los estudiantes, 
cuatro, ocho o doce horas, con diferentes lapsos de tiempo; el estudiante se acostumbra a producir dentro de estos límites. Los estudiantes con el pasar del tiempo adquieren una capacidad de reacción ante todas las situaciones en las que podrían estar sumergidos.

El estudiante está vinculado al profesor (A) por diferentes expectativas, especialmente su introducción a la práctica profesional. En este contexto, el estudiante elige un taller de proyectos tomando en cuenta dos tipos de cri- terios: la reputación de los profesionales que enseñan y el éxito profesional de los antiguos estudiantes del taller.

La interacción (B) relaciona al profesor y a los conocimientos de diseño que utiliza, por ejemplo, las herramientas profesionales (como arquitecto) y las referencias aconsejadas para el ejercicio (como un profesor). Frente a un ejercicio de diseño arquitectónico, el estudiante construye sus soluciones a partir del conocimiento adquirido, interacción (C), en sus cursos y en sus antiguos ejercicios.

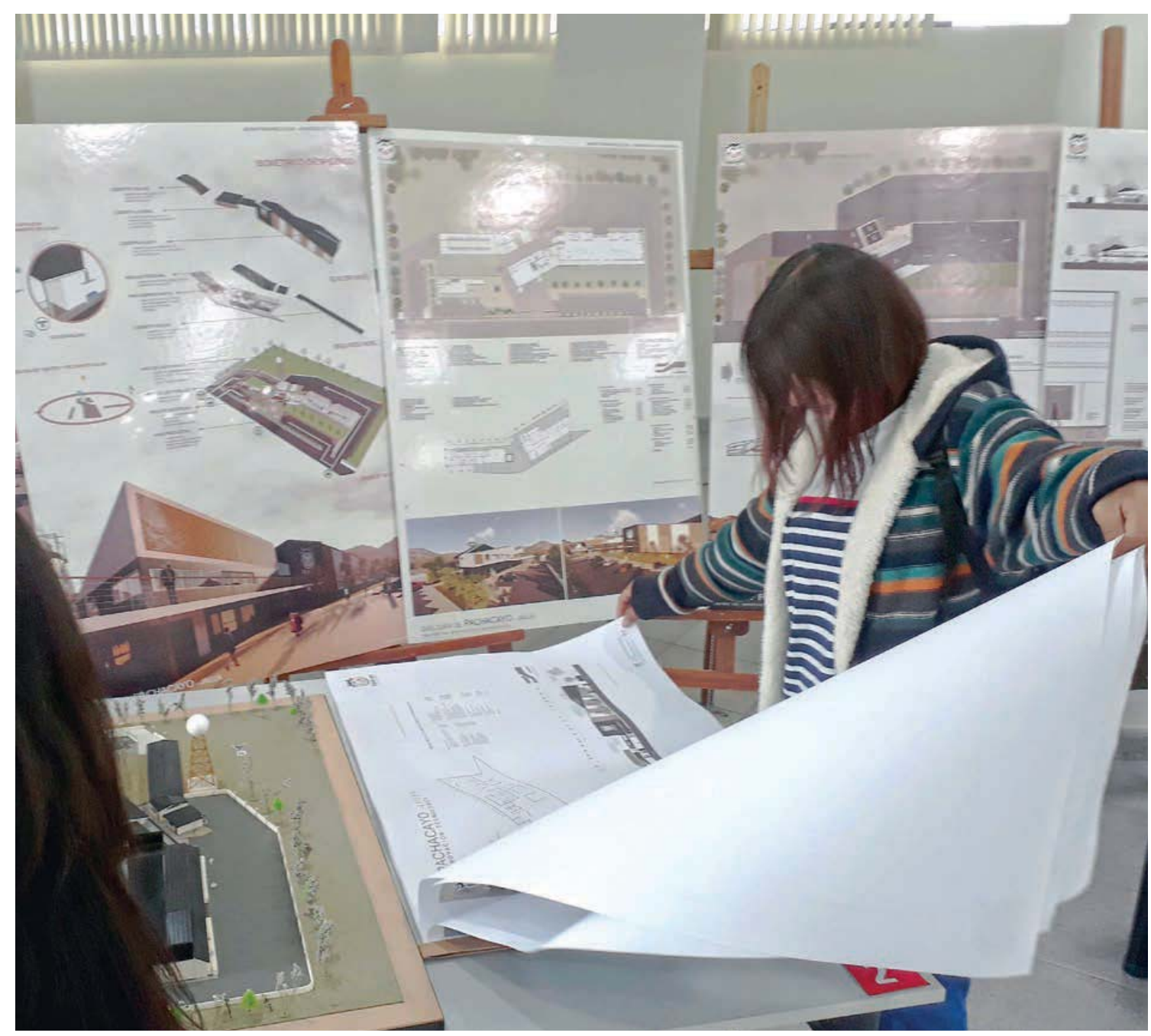

Figura 7. Estudiantes de arquitectura en la Expo-Arquitectura 2018-20.

Foto: Carol Alvarez Cortez

En resumen, se propone un ejercicio, en determinadas condiciones, a un estudiante; el profesor construye una situación artificial en la que se instala el estudiante para que llegue a un estado deseado de competencias, mediante la realización de tareas diseñadas para este fin. En otras palabras, el estudiante logra este estado deseado de competencias, de su actividad y de aquella manera gana experiencia. 


\section{Personalidad, maestro-estudiante en la enseñanza-aprendizaje}

En todos los campos de la educación, la personalidad del pedagogo "maestro" es de importancia decisiva. La enseñanza del proyecto arquitectónico exige, una y otra vez, una personalidad impregnada de cultura. El maestro es aquí también un educador $y$, como tal, no solo transmite conocimiento y experiencia, sino también despierta la conciencia de los hechos, de las cosas, de los estados, de los efectos, de las contradicciones y de la historia. El maestro da conciencia de las diferentes actitudes y juegos de reflexión, el maestro da conciencia del inconsciente como un rico alimento para el trabajo creativo y, finalmente, el maestro da conciencia de todos los sentidos del hombre de estas herramientas preciosas de nuestro trabajo. Dar conciencia es, probablemente, la contribución más importante del maestro durante el proceso de diseño arquitectónico, del proyecto, porque solo la asociación de los conocimientos aislados y de la conciencia de las relaciones de dependencia entre las cosas da nacimiento al poder crítico.

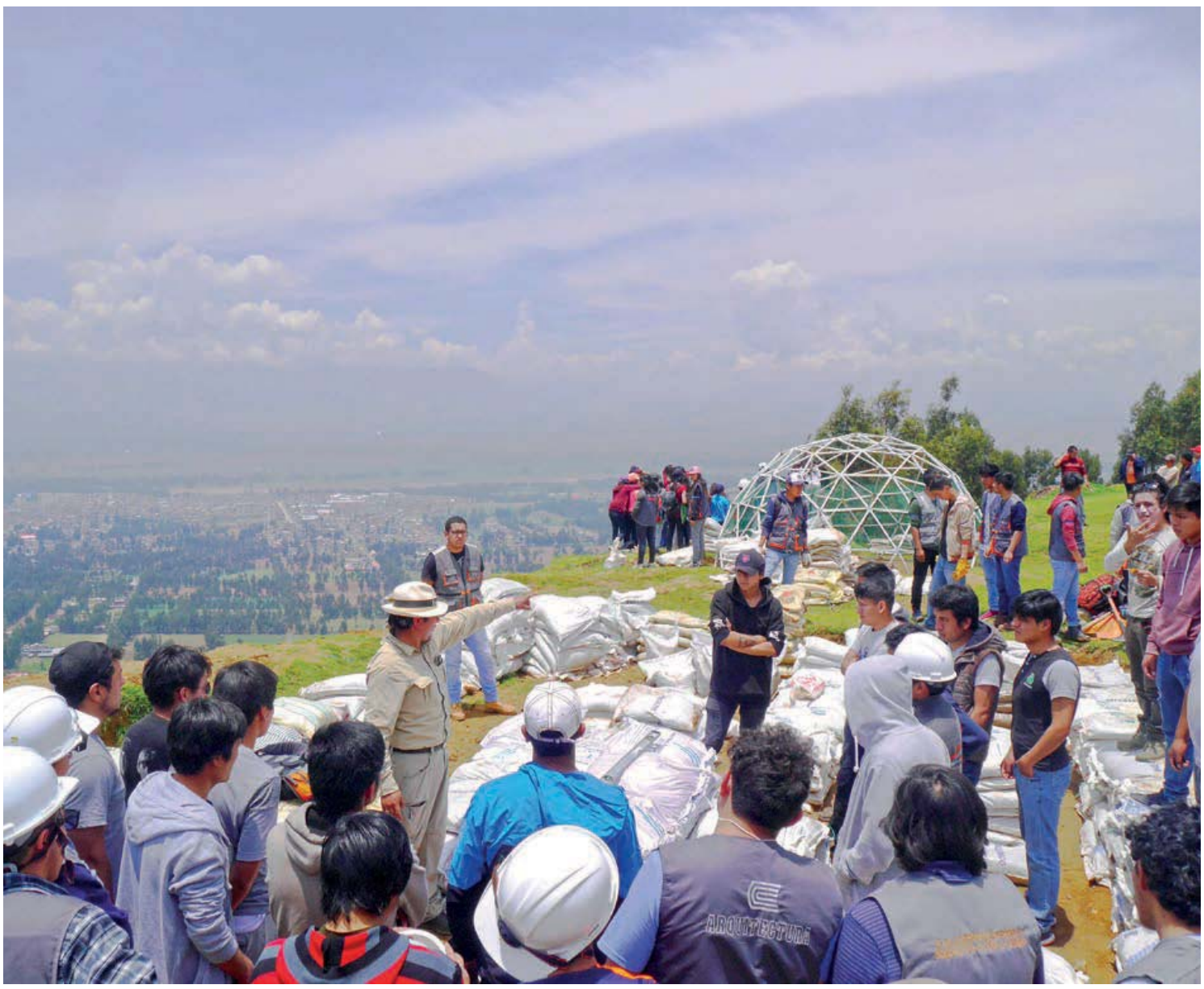

Figura 8. Workshop de Bioarquitectura. Semana Arquitectónica 2018-20 Foto: Carol Alvarez Cortez

La formación de la conciencia depende de la personalidad del estudiante, mucho más durante el proceso de diseño arquitectónico ("concepción») que durante la transmisión del conocimiento propiamente dicho. Desde un punto de vista orgánico, el estudiante es un adulto y tiene una experiencia vivida y un bagaje cultural; tiene una base de conocimiento y experiencia; tiene sus preferencias y antipatías, y a menudo conoce mejor sus propias habilidades que nosotros, los maestros. La huella de la familia, la escuela y la sociedad es fuerte, algunos rasgos de carácter son formados en sus grandes líneas. Por esta razón, la formación de la conciencia es 
una contribución al desarrollo de la personalidad. Pero, contrariamente a la transmisión de conocimiento, esta contribución no es una rama a revisar, esto no es posible porque la formación de la conciencia está profundamente arraigada en el individuo, tanto en la persona del maestro como en la del estudiante.

Personalmente, me tomó años descubrir la importancia de la personalidad del individuo y la formación de la conciencia. Primero, acepté el desafío vida académica; luego, cuando tuve a un grupo de estudiantes, que estaban listos para aprender y concebir un proyecto arquitectónico, traté de enseñar más o menos directamente desde mi experiencia profesional. Pero, como los estudiantes están en formación, me vi obligado a reducir mis exigencias cada vez más y a simplificar sin interrumpir mi forma de transmitir el oficio. Sobre la base de la experiencia adquirida durante el período inicial de la enseñanza universitaria, gradualmente logré construir una manera de enseñanza que -creo yo- hoy se adapta a mi persona y corresponde a mis ideas sobre la formación de la personalidad y a la educación (enseñanzaaprendizaje) en arquitectura.

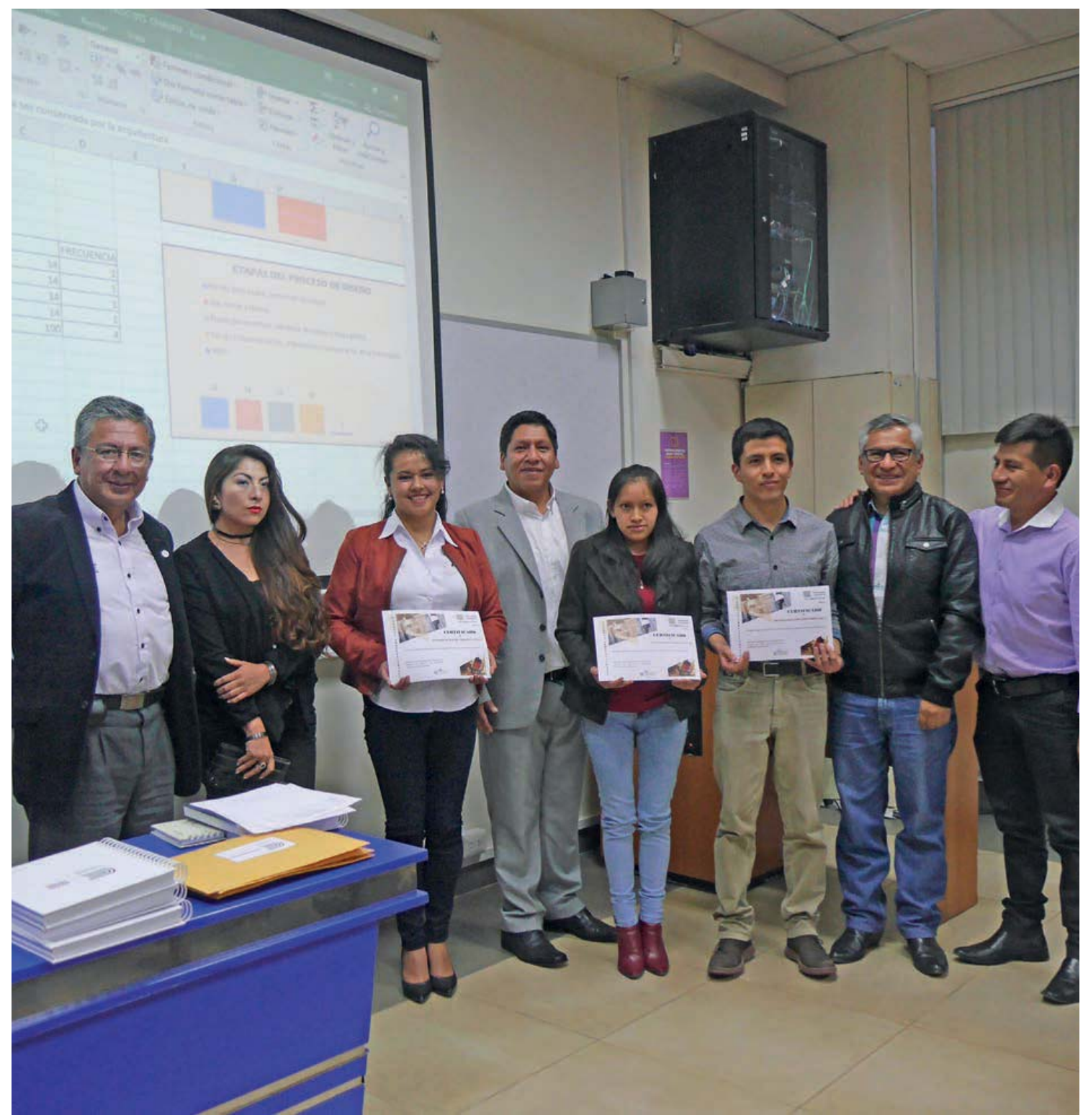

Figura 9. Conversatorio docente y premiación de la Expo-Arquitectura 2018-20 Foto: Carol Alvarez Cortez 


\section{La triada: estudiante, proyecto y maestro}

La arquitectura está hecha de "relaciones»; la naturaleza humana, por el contrario, durante la elaboración de un proyecto, procede linealmente, especialmente en un estudiante que todavía tiene poca práctica. Por este motivo, el trabajo del proyecto debe dividirse en diferentes fases y estas están íntimamente conectadas. Por ello el maestro siempre tiene que hablar de relaciones entre las cosas. Pero si esto se hace en teoría, entonces el enfoque no está lo suficientemente vivo. Así que, personalmente, pido a cada estudiante, al comienzo de cualquier trabajo y en muy poco tiempo, un proyecto en forma de boceto. Con la ayuda de este boceto, que tiene la ventaja de presentar muchas fallas, es más fácil hablar sobre los problemas, sus elementos y sus interrelaciones. Hablar demasiado o poco tiempo sobre estas interrelaciones puede bloquear la creatividad. Y lleva al estudiante a creer que "concebir" es resolver los problemas, uno tras otro. No obstante el diseño requiere ambas cosas: una visión general de lo que es posible y probable y el trabajo de detalle.

La construcción, durante el trabajo de elaboración, no es solo un ejercicio adicional, permite, un trabajo simultáneo de vista general y detalle para aprender los principios de construcción y mejorar el proyecto al mismo tiempo, por ejemplo, las fachadas.

La enseñanza de la concepción y el diseño en sí mismo incluye innumerables elementos opuestos que parecen contradecirse entre sí. Una de estas contradicciones es la "voluntad del creador" que se opone a la "voluntad de su obra". Sin la voluntad del creador frente a una obra dada, la concepción no es posible... El estudiante debe elegir el sentido de imagen y el concepto de una obra, así como un principio de organización espacial. Debe traer la voluntad de dar forma antes de comenzar y durante el trabajo. Pero, a medida que el trabajo progresa, el trabajo creado manifiesta cada vez más su propia voluntad. Las normas que el creador le ha dado, las propiedades, las contradicciones, las cualidades y los defectos de la obra; no se requiere, entonces, solo la voluntad de la persona que lo creo, sino también el diálogo de esta con su obra. Esta obra es como su propio hijo, quien, llegado a la madurez, plantea sus propios requisitos, quien manifiesta su propia "personalidad", que luego debe ser aceptada. Los estudiantes, hombres y mujeres, a menudo me miran avergonzados cuando les hablo de su "hijo", el hijo al que dieron a luz. El estudiante y su obra están obligados a dialogar. El trabajo habla a través del dibujo. Cuanto más preciso y completo sea el dibujo, más claro será su lenguaje; él responde a nuestras preguntas, usa nuestro conocimiento y nuestros sentidos.

El diálogo con la obra no solo es atractivo para nuestro intelecto (nuestro conocimiento teórico), sino también para nuestros sentidos (para nuestra sensibilidad e imaginación). Cuando ambos lados del poder creativo tienen un cierto equilibrio, la posibilidad de ser un buen arquitecto aumenta. Sin embargo, las capacidades intelectuales y sensoriales no siempre juegan un papel igual. En tal caso, el creador decide en qué casos debe usar más sus capacidades intelectuales y en qué otros casos, sus capacidades sensoriales. Estas habilidades y capacidades son como instrumentos que deberían estar disponibles para nosotros como deseamos. Es probable que sea la manera del manipular lo que hace que el talento de un buen arquitecto.

El asesoramiento a los estudiantes, comúnmente llamado crítica, nos permite a los maestros dialogar en varias reuniones con todos los estudiantes, en la mesa de trabajo, para lo cual dedicamos un tiempo prudente. En realidad, recordemos que somos tres en la mesa: el estudiante, el maestro y el "hijo" del estudiante; podríamos pensar que es una discusión bidireccional, pero es una discusión tripartita. Aunque el proyecto y su autor constituyen una unidad. La crítica en la mesa de trabajo nos sumerge en un mundo de dimensiones cósmicas. El primer asesoramiento al estudiante, usualmente, ocurre cuando se dan los primeros ejercicios (esquisses). La crítica comienza con el contenido de la propuesta. En general, el estudiante explica sus intenciones y sobre la imagen que tiene del trabajo terminado. La propuesta inicial muestra el enfoque vacilante de los problemas inherentes a la tarea dada, la crítica va desde la discusión del proyecto global a los procesos, y luego vuelve al proyecto global; este diálogo con el estudiante revela, en todos los casos, puntos a mejorar en la propuesta hecha por el estudiante (aprendiz en formación). Debo confesar que no dejo de sorprenderme cada vez que veo y constato en qué medida 
el entorno cultural del estudiante influye en su proyecto. El carácter del proyecto de un estudiante de la sierra difiere en general del de un estudiante de la selva, el de la selva es diferente al de la costa. Es impresionante ver hasta qué punto los estudiantes están inmersos en la cultura propia de sus localidades de origen y su entorno inmediato, $y$, por ende, estos ya tienen una rica cultura arquitectónica.

Finalmente, debo señalar que el proceso de aprendizaje de un maestro también se realiza a través de los estudiantes; es uno de los estímulos más importantes para cualquier reflexión pedagógica. Generaciones de estudiantes pasan a una alta velocidad. Con ellos cambian el saber, la curiosidad y los modelos. Para el maestro, estos cambios representan nuevos retos. También debe haber un proceso de aprendizaje entre los profesores.

La discusión sobre la arquitectura entre profesores es necesaria, no para eliminar las contradicciones, sino para aclarar las opiniones y aprender reciprocamente. Una discusión, sin duda alguna, es más estimulante si se hace de manera clara y abierta porque promueve el respeto del interlocutor, así como las reflexiones.
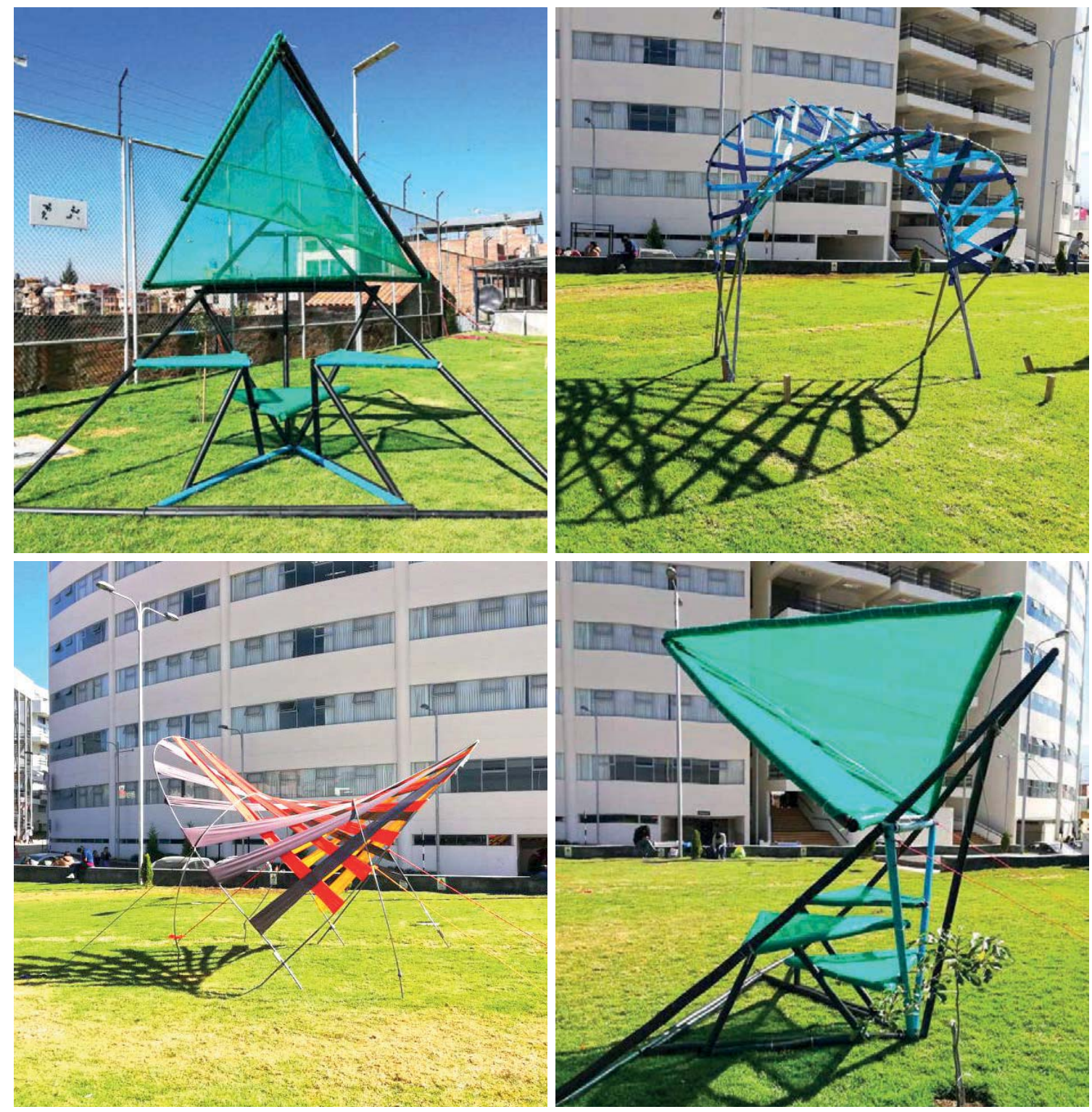

Figura 10. Experimentación de los estudiantes del curso Estructuras, docente Arq. Leonardo Casas. Fotos: Archivo de la Escuela Académico Profesional de Arquitectura 


\section{EL PROYECTO ARQUITECTÓNICO COMO OBJETO DE LA INVESTIGACIÓN CIENTÍFICA EN ARQUITECTURA}

La investigación en la disciplina arquitectónica está íntimamente vinculada al enfoque del proyecto, que tiene una dimensión intrínsecamente interpretativa. De hecho, las dimensiones de las posibilidades de un proyecto son extremadamente variadas; el proyecto, al proponer una multitud de contingencias, crea una brecha entre la proyección y la realización, un intersticio donde el trabajo del arquitecto investigador encuentra su lugar.

En una primera parte de esta reflexión, nos centraremos en el lugar del proyecto en la investigación en arquitectura con un enfoque esencialmente bibliográfico. Tenemos en cuenta que estos temas no son nuevos y ya han sido abordados por algunos autores y en diferentes contextos nacionales y, sobre todo, internacionales. En una segunda parte, elegimos inscribirnos en el marco de la enseñanza y la investigación en arquitectura, basándonos en los temas de trabajo de arquitectura productiva, desarrollados por los estudiantes de la Escuela Académico Profesional Arquitectura de la Universidad Continental, y en los campos disciplinarios de la enseñanza de la arquitectura se dará una clasificación de la investigación en arquitectura a través del proyecto.

De manera transversal, nos parece importante cuestionar tanto sobre la práctica de la investigación en arquitectura en sí misma, como sobre los aspectos de la práctica docente en arquitectura. En principio, es a partir de esta práctica que se puede tejer "una tradición de investigación arquitectónica», tal como existe en otras disciplinas académicas, una tradición en todo lo que tiene de la vida (transmisión de conocimiento, conocimiento, métodos, problemas, de una generación a otra).

Es por ello que, desde los ambientes académicos, reflexionamos sobre la ubicación del proyecto en la investigación científica en arquitectura y tratamos de responder las interrogantes siguientes: ¿Qué es la investigación arquitectónica?, ¿hasta qué punto la investigación en arquitectura ayuda a producir arquitectura? Desde el punto de vista epistemológico, la arquitectura es una disciplina práctica (fundamentada en la praxis), es decir, está orientada hacia la acción, la transformación del mundo y, por lo tanto, hacia el proyecto. Proponemos aquí la hipótesis de que la transacción entre "saber" $\mathrm{y}$ "hacer" se realiza mediante el proyecto y el lugar más o menos importante que se le otorga al proyecto dentro de las investigaciones en arquitectura.

El enfoque de diseño (concepción) trata sobre la investigación por y para el proyecto. De hecho, esta relación entre la investigación y el proyecto ha sido abordada por otros autores y no siempre tiene los mismos significados.

Esta reflexión se centra fundamentalmente en el contexto de trabajos de fin de carrera presentados en el periodo 2018. Nuestro campo de estudio, por lo tanto, se focaliza en el trabajo de investigación en arquitectura dentro de la educación superior y, más precisamente, en nuestra universidad. Es importante destacar que nos centraremos solo en el alcance de la investigación en arquitectura a nivel de pregrado y no en toda la investigación en arquitectura. Antes de continuar con nuestra reflexión, sobre el lugar y la definición del proyecto, parece necesario aclarar el contexto en el que está incrustado nuestro enfoque de la arquitectura, el cual se desarrolla, en particular, la investigación por y para el proyecto.

Para comprender el significado del concepto del proyecto y la importancia que se le otorga en la investigación en arquitectura, primero, volveremos al carácter polisémico del término del proyecto, poniendo énfasis en las características del proyecto arquitectónico y urbano. En una segunda parte, veremos una clasificación según los diferentes enfoques del proyecto.

\section{Definiciones y conceptos del proyecto}

Dado que nos preguntamos sobre el lugar del proyecto en la investigación arquitectónica, primero queremos especificar el término del proyecto al que nos referimos en esta publicación.

La definición del término proyecto tiene su origen en la palabra latina proiectus, cuyo significado es planificación, que consiste en un conjunto de actividades que se encuentran interrelacionadas y coordinadas. Según el Diccionario de la 
Real Academia de la Lengua Española, en su tercera y cuarta acepciones, respectivamente, proyecto es el "designio o pensamiento de ejecutar algo", y el "conjunto de escritos, cálculos y dibujos que se hacen para dar idea de cómo ha de ser y lo que ha de costar una obra de arquitectura o de ingeniería».

En gestión, el término "proyecto" se refiere a la organización social para un propósito y tiempo específico (Boltanski \& Chiapello, 1999), aquí se hace referencia a los paradigmas de red y comunicación. En el campo de las ciencias humanas y sociales, Jean Pierre Boutinet exploró la noción de proyecto, según él, es una de las figuras características de la modernidad por el entrelazamiento de espacio y tiempo que esta implica. Entonces - para el autor- la noción del proyecto como concepto se refiere "a los objetos que la modernidad cultiva" y como figura, a la "capacidad de crear" por individuos y colectivos.

Boutinet (2012) afirma que el proyecto arquitectónico representa "el paradigma de cualquier proyecto". El proyecto es un proceso en el que se lleva a cabo el trabajo de "Diseño". Esto abarca la actividad profesional de los arquitectos, las formas de pensamiento en el marco de esta práctica y los resultados materiales, tangibles, de esta actividad (Conan, 1990). La concepción es el proceso que conduce a la invención de elementos físicos que, en respuesta a una función, deben realizarse; proponen un nuevo orden físico, una nueva organización, una nueva forma (Alexander, 1971).

Jean Blanc (2007) dice que el proyecto arquitectónico convierte la reflexión en realidad al hacer una pregunta inesperada que renueva su aprensión; este concepto es un tipo de enfoque intelectual, el "proyecto pensado", según sus términos, propone una posible construcción del mundo. "Proyectar no es solo crear algo nuevo. Se trata de crear un todo, agregar algo nuevo a algo que ya existe. Cada proyecto debe estar entre lo existente y lo que aún no existe» (Nelson \& Stolterman, 2003). El proyecto es un "pasaje» entre la demanda social y el espacio arquitectónico (Prost, 2014).

Históricamente, el proyecto urbano difiere del proyecto de arquitectura, pero hoy parece que estos dos modelos tienden a acercarse. El proyecto urbano agrega otras dimensiones a la no- ción de proyecto. El proyecto urbano debe tener en cuenta otros proyectos que se despliegan en varias escalas para construir la ciudad. "Ya no pensamos en la ciudad en partes, sino en su conjunto, un sistema relacional, un lugar que permite la incorporación de diferentes tejidos urbanos y sociales» (Ingallina \& Roncayolo, 2010).

Manuel Castells define el proyecto como la expresión de una idea; distingue al proyecto del programa, que le traduce la idea con más detalle. Esta transacción entre el proyecto y la planificación urbana está permitida por la implementación de una estrategia, la mayoría de las veces es llevada a cabo por el organismo politico. Es quizás esta entrada estratégica la que le da al proyecto urbano toda su especificidad. Quizá también la propiedad de elaboración colectiva del proyecto urbano sea también parte de estas especificidades (Castells, 1983). El proyecto urbano es, entonces, una elaboración colectiva en torno a un sistema de intenciones, un objetivo estratégico en un territorio y un diseño espacial.

Para Paola Vigano (2015), el «proyecto de ciudad y territorio" es "productor de conocimiento". Es un dispositivo cognitivo para explorar el contexto espacial, "el proyecto es una forma de estudio e investigación». Vigano está realizando un estudio sobre el estado epistemológico del proyecto y brinda argumentos a favor de "la investigación sobre el proyecto como una herramienta para pensar y producir conocimiento". Esta investigación produce ideas que enriquecerán el debate sobre el territorio e interrogarán desde el interior del proceso a la toma de decisiones para identificar pistas relevantes para las construcciones del avenir.

En esta reflexión, cuando se habla del término proyecto, nos referiremos al proyecto arquitectónico y urbano y cuestionaremos su lugar en la investigación en arquitectura.

\section{Relación del proyecto e investigación en arquitectura}

Basado en los trabajos de los estudiantes y los textos especializados referentes sobre el tema y, particularmente, en la división de la investigación en diseño propuesta por Christopher Frayling (1993): la investigación para diseño, sobre el diseño y por el diseño. El autor parte de la 
suposición que la investigación arquitectónica aborda el proyecto desde diferentes ángulos y entradas diferentes, por lo tanto, proponemos distinguir la investigación en:

- Investigación por el proyecto: se utiliza el proyecto como herramienta de investigación para experimentar con proposiciones, revelar situaciones y recopilar discursos en una relación directa con la realidad.

- Investigación sobre el proyecto: estudia las representaciones y las realizaciones concretas como manifestaciones del proyecto, un corpus de proyectos es el apoyo de la investigación.

- Investigación para el proyecto: aprovecha el proyecto desde dentro, describe las relaciones entre los actores del mismo proyecto, analiza su modo de producción, realiza una "ecología" del proyecto.

Basado en mi experiencia profesional y académica, puedo afirmar que estas categorias de enfoque del proyecto en la investigación no son exclusivas, por lo tanto, el trabajo de investigación puede cubrir dos o más enfoques diferentes del proyecto.

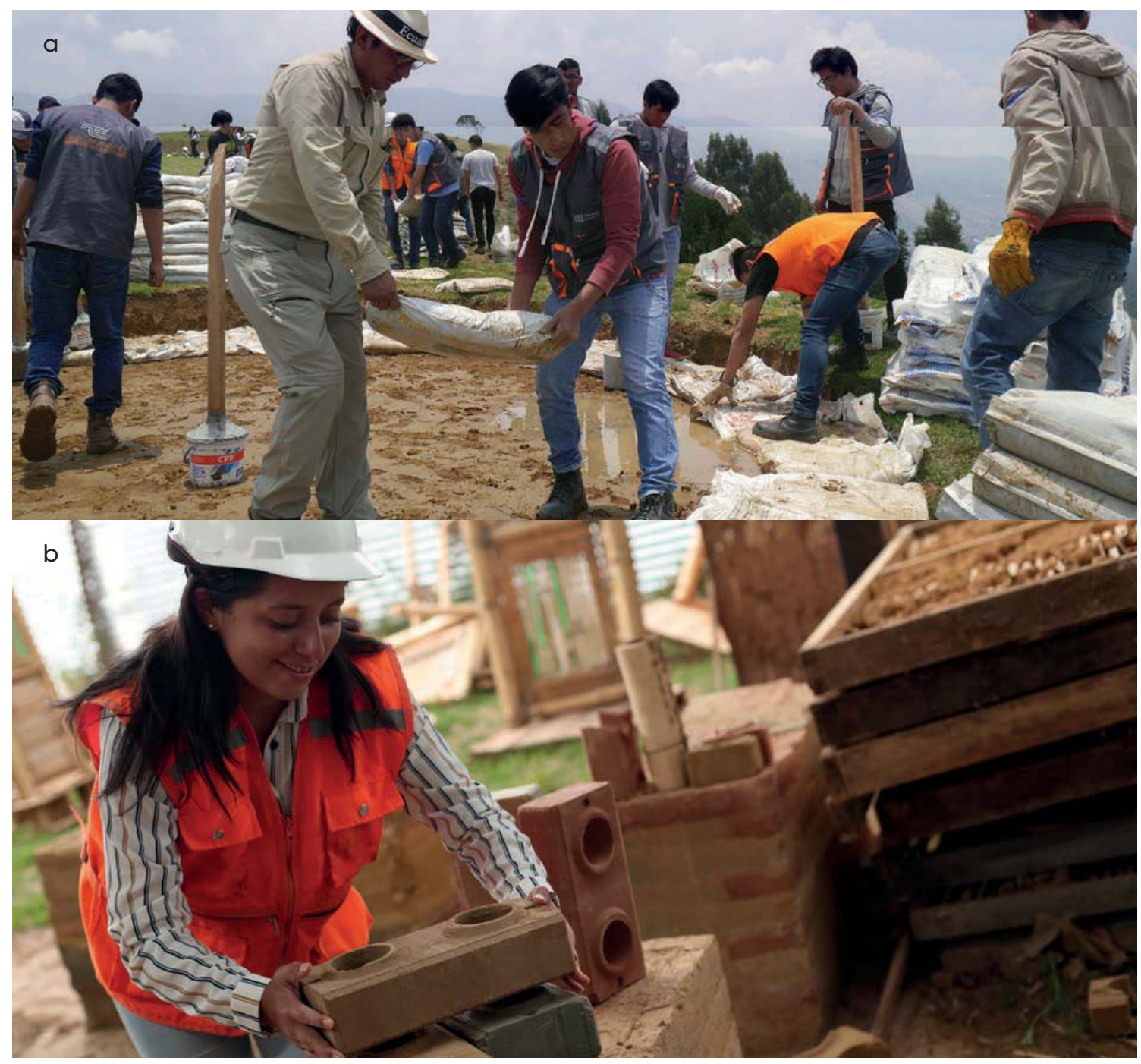

Figura 11. Trabajos conducidos por el Laboratorio en tierra

a. Arq. Vladimir Montoya en el Workshop de Bioarquitectura 2018-20

Foto: Carol Alvarez

b. Pamela Mallma, egresada y docente de la EAP Arquitectura

Foto: Comunicaciones UC 


\section{BIBLIOGRAFÍA}

Alexander, C. (1971). De la synthèse de la forme: essai. Paris, France: D Dunod.

Blanc, J. N. (2007). Le projet et la "pensée projet". Saint-Etienne, France: Publications de l'Université de Saint Etienne.

Boltanski, L. \& Chiapello. É. (1999). Le nouvel esprit du capitalisme. París, France: Gallimard.

Boutinet. J. P. (2012). Anthropologie du projet. París, France: Presses Universitaires de France.

Castells. M. (1983). The city and the grassroots: A cross cultural theory of urban social movements. California, EE. UU.: University of California Press.

Conan, M. (1990). Concevoir un projet d'architecture. Paris, France: I'Hannattan.

Frayling, C. (1993). Research in art and design. London: Royal College of Art.

Ingallina, P. \& Roncayolo, M. (2010). Le projet urbain. París, France: Presses Universitaires de France.

Lebahar, J. (2001). "A didactic approach to architecture design education: comparative study of two situations". Didaskalia n. ${ }^{\circ} 19$ pp. 39-77

Johsua, S. \& Dupin J.J. (1993). Introduction à la didactique des sciences et des mathématiques. París, France: PUF.

Nelson, H. G. \& Stolterman. E. (2003). The design way: intentional change in an unpredictable world : foundafions and fundamentals of design competence. Englewood Cliffs. N. J.: Educational Technology Publications.

Prost, R. (2014). Pratiques de projet en architecture: le tournant silencieux. Gollion. Suisse: Infolio.

Viganò. P. (2015). «Le probléme du projet comme producteur de connaissance". En re_ARCH'Y: en architecture, la recherche et le projet (ENSAL et ULPGC. pp. 60-64). Lyon (France) et Las Palmas (Espagne). 